\title{
Toxoplasma gondii Infection and Mixed Anxiety and Depressive Disorder: A Case-Control Seroprevalence Study in Durango, Mexico
}

\author{
Cosme Alvarado-Esquivel ${ }^{\mathrm{a}, \mathrm{h}}$, Luis Francisco Sanchez-Anguiano ${ }^{\mathrm{b}}$, Jesus Hernandez-Tinoco ${ }^{\mathrm{b}}$, \\ Luis Omar Berumen-Segovia ${ }^{a}$, Yazmin Elizabeth Torres-Prieto ${ }^{c}$, Sergio Estrada-Martinez ${ }^{b}$, \\ Alma Rosa Perez-Alamos ${ }^{\text {b }}$, Maria Nalleli Ortiz-Jurado ${ }^{d}$, Gabriel Molotla-de-Leon ${ }^{d}$, \\ Isabel Beristain Garciac ${ }^{c}$, Elizabeth Rabago-Sanchez ${ }^{\mathrm{a}, ~}$, Oliver Liesenfeld ${ }^{\mathrm{f}, \mathrm{g}}$
}

\begin{abstract}
Background: The parasite Toxoplasma gondii (T. gondii) may invade the brain and might induce behavioral changes. We sought to determine the association of $T$. gondii infection and mixed anxiety and depressive disorder.
\end{abstract}

Methods: Through an age- and gender-matched case-control seroprevalence study, we examined 65 patients suffering from mixed anxiety and depressive disorder (WHO ICD-10 code: F41.2) attending in a public hospital of mental health and 260 control subjects without this disorder from the general population. Sera of participants were analyzed for anti-Toxoplasma $\operatorname{IgG}$ and IgM antibodies using enzymelinked immunoassays.

Results: Fifteen $(23.1 \%)$ of the 65 patients and 18 (6.9\%) of the 260 controls had anti-T. gondii $\operatorname{IgG}$ antibodies (odds ratio (OR): 4.03; 95\% confidence interval $(\mathrm{CI}): 1.90-8.53 ; \mathrm{P}<0.001)$. The frequency of high ( $>150 \mathrm{IU} / \mathrm{mL})$ anti-T. gondii IgG levels was similar in cases and controls (OR: 0.25; 95\% CI: $0.05-1.06$; P $=0.05$ ). Seroprevalence was similar in male cases and controls $(\mathrm{P}=1.0)$; however, se-

Manuscript accepted for publication May 03, 2016

${ }^{a}$ Faculty of Medicine and Nutrition, Juarez University of Durango State, Avenida Universidad S/N, 34000 Durango, Durango, Mexico

bInstitute for Scientific Research "Dr. Roberto Rivera Damm", Juarez University of Durango State, Avenida Universidad S/N, 34000 Durango, Durango, Mexico

'Facultad de Enfermeria y Obstetricia, Juarez University of Durango State, Cuauhtemoc 223 Norte, 34000 Durango, Mexico

dHospital of Mental Health "Dr. Miguel Vallebueno", Servicios de Salud de Durango, Durango, Mexico

${ }^{\text {e}}$ General Hospital, Secretary of Health, Avenida 5 de febrero 220, $34000 \mathrm{Du}-$ rango, Mexico

fInstitute for Microbiology and Hygiene, Campus Benjamin Franklin, Charite Medical School, Hindenburgdamm 27, D-12203 Berlin, Germany

gCurrent address: Roche Molecular Systems, Pleasanton, CA 94588, USA

${ }^{\mathrm{h}}$ Corresponding Author: Cosme Alvarado-Esquivel, Laboratorio de Investigacion Biomedica, Facultad de Medicina y Nutricion, Avenida Universidad S/N, 34000 Durango, Dgo, Mexico. Email: alvaradocosme@yahoo.com

doi: http://dx.doi.org/10.14740/jocmr2576w roprevalence was significantly higher in female cases than in female controls (OR: 7.08; 95\% CI: 2.83 - 17.67; P $<0.00001$ ). Patients aged 31 - 50 years old had a significantly higher seroprevalence of $T$. gondii infection than controls of the same age group (OR: 21.04; $95 \%$ CI: 5.22 - 84.80; P < 0.00001). Anti-T. gondii IgM antibodies were found in four $(26.7 \%)$ of the 15 anti-T. gondii IgG seropositive cases and in $10(55.6 \%)$ of the 18 anti-T. gondii IgG seropositive controls $(\mathrm{P}=0.15)$.

Conclusions: Results support for the first time an association between seropositivity to $T$. gondii and mixed anxiety and depressive disorder. Further research to confirm this association and to determine the seroepidemiology of $T$. gondii infection in patients with this disorder is needed.

Keywords: Toxoplasma gondii; Seroprevalence; Mixed anxiety and depressive; Psychiatric patients; Case-control

\section{Introduction}

Toxoplasma gondii (T. gondii) is a coccidian parasite causing infections worldwide [1]. About $30 \%$ of humanity is currently infected with $T$. gondii [2]. Infection with this parasite is usually acquired by ingestion of raw or undercooked meat containing tissue cysts, and water or food contaminated with oocysts shed by cats [3]. Other routes of T. gondii infection include congenital transmission [4], organ transplantation [5], and blood transfusion [6]. Although infections with T. gondii are usually asymptomatic, some infected individuals develop a symptomatic disease known as toxoplasmosis with involvement of lymph nodes, central nervous system or eyes $[2,7,8]$. Some psychiatric disorders have been associated with infection with $T$. gondii, i.e. schizophrenia $[9,10]$, bipolar disorder [11], obsessive compulsive disorder [11, 12], depression [13], and generalized anxiety disorder [14]. However, to the best of our knowledge, the association of infection with $T$. gondii and mixed anxiety and depressive disorder has not been reported. In this disorder, with the code F41.2 according to the classification of mental and behavioral disorders of the ICD-10 (http:// 
Table 1. Comparison of Seropositivity Rate to T. gondii According to Sex and Age Between Cases (F41.2) and Controls

\begin{tabular}{|c|c|c|c|c|c|c|c|}
\hline \multirow{3}{*}{ Characteristics } & \multicolumn{3}{|c|}{ Cases } & \multicolumn{3}{|c|}{ Controls } & \multirow{3}{*}{ P value } \\
\hline & \multirow{2}{*}{ No. tested } & \multicolumn{2}{|c|}{ Seroprevalence of $T$. gondii infection } & \multirow{2}{*}{ No. tested } & \multicolumn{2}{|c|}{ Seroprevalence of $T$. gondii infection } & \\
\hline & & No. & $\%$ & & No. & $\%$ & \\
\hline Male & 23 & 2 & 8.7 & 92 & 8 & 8.7 & 1.0 \\
\hline Female & 42 & 13 & 31.0 & 168 & 10 & 6.0 & $<0.0001$ \\
\hline 30 or less & 24 & 4 & 16.7 & 96 & 7 & 7.3 & 0.2 \\
\hline $31-50$ & 26 & 10 & 38.5 & 104 & 3 & 2.9 & $<0.0001$ \\
\hline$>50$ & 15 & 1 & 6.7 & 60 & 8 & 13.3 & 0.7 \\
\hline
\end{tabular}

apps.who.int/classifications/icd10/browse/2016/en\#/F40F48), anxiety and depression are both present in an individual, but neither is predominant, and neither symptom is present to an extent that justifies a separated diagnosis. Given the lack of information about the link of infection with $T$. gondii and this specific diagnosis, we attempted to determine the association between $T$. gondii infection and mixed anxiety and depressive disorder in a sample of patients attending in a public hospital of mental health in the northern Mexican city of Durango.

\section{Materials and Methods}

\section{Study design and populations studied}

We performed an age- and gender-matched case-control study of 65 psychiatric patients suffering from mixed anxiety and depressive disorder attending in a public hospital of mental health in Durango City, Mexico and 260 control subjects without mixed anxiety and depressive disorder from the general population of the same city. This case-control study was performed from August 2015 to February 2016. Inclusion criteria for cases were: 1) patients suffering from mixed anxiety and depressive disorder attending in the Hospital of Mental Health "Dr. Miguel Vallebueno" of the Secretary of Health in Durango City; 2) aged 18 years and older; and 3) who accepted to participate in the study. Diagnosis of mixed anxiety and depressive disorder was made by psychiatrists and was classified as ICD-10 code F41.2. Of the 65 cases, 42 (64.6\%) were females and $22(35.4 \%)$ were males. Mean age in cases was $39.43 \pm 14.05$ (range 18 - 77) years old. Control subjects were randomly selected from the general population of Durango City and matched with cases for age ( \pm 3 years) and gender. Inclusion criteria for controls were: 1) subjects of the general population of Durango City without anxiety and depression; 2 ) aged 18 years and older; and 3 ) who accepted to participate in the study. Of the 260 controls, $168(64.6 \%)$ were females and $92(35.4 \%)$ were males. Mean age in controls was $39.45 \pm$ 13.98 (range 18 - 78) years old. No statistically significant differences in age $(\mathrm{P}=0.99)$ and gender $(\mathrm{P}=1.0)$ between cases and controls were found.

\section{Detection of anti-T. gondii IgG and IgM antibodies}

A blood sample from each participant was collected. Blood samples were centrifuged and serum samples were obtained and stored at $-20{ }^{\circ} \mathrm{C}$ until analyzed. Sera were analyzed for anti- $T$. gondii $\mathrm{IgG}$ antibodies with the commercially available enzyme immunoassay (EIA) kit "Toxoplasma IgG" (Diagnostic Automation/Cortez Diagnostics Inc., Woodland Hills, CA, USA). A cut-off of $\geq 8$ International Units (IU) $/ \mathrm{mL}$ of anti- $T$. gondii IgG antibody was used. Sera of patients seropositive for anti-T. gondii IgG antibodies were further analyzed for antiT. gondii IgM antibodies by the commercially available EIA "Toxoplasma IgM" kit (Diagnostic Automation/Cortez Diagnostics Inc.). All IgG and IgM assays were performed following the manufacturer's instructions.

\section{Statistical analysis}

We used the software Epi Info version 7 and the SPSS version 15.0 (SPSS Inc. Chicago, IL) to perform the statistical analysis. For calculation of the sample size, we used a $95 \%$ confidence interval (CI) level, a power of $80 \%$, a 1:4 proportion of cases and controls, a reference seroprevalence of $6.1 \%$ [15] as the expected frequency of exposure in controls, and an odds ratio (OR) of 3.5. The result of the sample size calculation was 60 cases and 237 controls. We used the Student's $t$-test to compare the age among cases and controls. The association of $T$. gondii infection and mixed anxiety and depressive disorder was assessed with the two-tailed Pearson's Chi-squared test. OR and 95\% CI were calculated, and a $\mathrm{P}$ value $<0.05$ was considered as statistically significant.

\section{Ethical aspects}

The Ethics Committee of the General Hospital of the Secretary of Health in Durango City, Mexico approved this project. The purpose and procedures of this study were explained to all participants. In addition, a written informed consent was obtained from each participant. 


\section{Results}

Fifteen $(23.1 \%)$ of the 65 patients with mixed anxiety and depressive disorder and $18(6.9 \%)$ of the 260 controls had anti- $T$. gondii IgG antibodies. The difference in seroprevalence of $T$. gondii infection between cases and controls was statistically significant (OR: 4.03; 95\% CI: 1.90 - 8.53; P $<0.001$ ). Of the 15 anti-T. gondii IgG positive cases, five $(33.3 \%)$ had IgG levels higher than $150 \mathrm{IU} / \mathrm{mL}$, one $(6.7 \%)$ between 100 and 150 $\mathrm{IU} / \mathrm{mL}$, and nine $(60.0 \%)$ between 8 and $99 \mathrm{IU} / \mathrm{mL}$. In contrast, of the 18 anti-T. gondii IgG positive controls, $12(66.7 \%)$ had IgG levels higher than $150 \mathrm{IU} / \mathrm{mL}$, one $(5.5 \%)$ between 100 and $150 \mathrm{IU} / \mathrm{mL}$, and five $(27.8 \%)$ between 8 and $99 \mathrm{IU} /$ $\mathrm{mL}$. The frequency of high ( $>150 \mathrm{IU} / \mathrm{mL})$ anti-T. gondii $\mathrm{IgG}$ levels was similar in cases and controls (OR: 0.25; 95\% CI: 0.05 - 1.06; $\mathrm{P}=0.05)$.

Seroprevalence of $T$. gondii infection was stratified by sex and age (Table 1). We observed that seroprevalence was similar in male cases and male controls $(\mathrm{P}=1.0)$; however, seroprevalence was significantly higher in female cases than in female controls (OR: 7.08; 95\% CI: 2.83 - 17.67; P < 0.00001). Patients aged 31 - 50 years old had a significantly higher seroprevalence of $T$. gondii infection than controls of the same age group (OR: 21.04; 95\% CI: 5.22 - 84.80; P < 0.00001). Seroprevalence did not vary between cases and controls in the age groups of 30 years and younger, and older than 50 years.

With respect to IgM seropositivity, anti-T. gondii IgM antibodies were found in four $(26.7 \%)$ of the 15 anti- $T$. gondii IgG seropositive cases and in $10(55.6 \%)$ of the 18 anti-T. gondii IgG seropositive controls. No statistically significant difference in the frequency of IgM seropositivity among cases and controls was found $(\mathrm{P}=0.15)$.

\section{Discussion}

The association of $T$. gondii infection and mixed anxiety and depressive disorder is largely unknown. This case-control seroprevalence study aimed to determine whether $T$. gondii seropositivity is associated with mixed anxiety and depressive disorder in Durango City, Mexico. Results of the present study show that psychiatric patients suffering from mixed anxiety and depressive disorder have a significantly higher frequency of anti- $T$. gondii IgG antibodies than age- and gender-matched control subjects without this disorder of the same city. Remarkably, the seroprevalences found in women $(31.0 \%)$ and patients aged 31 - 50 years old $(38.5 \%)$ suffering from mixed anxiety and depressive disorder in our study are very high in Durango City, compared with the $6.1 \%$ seroprevalence of $T$. gondii exposure reported in the general population in the same city [15]. To the best of our knowledge, this is the first report of an association of $T$. gondii infection and mixed anxiety and depressive disorder. There are some reports of the association of $T$. gondii infection with either anxiety or depression but not about the association of infection with the presence of both anxiety and depression in the same patients. In a recent study, generalized anxiety disorder was associated with both seroprevalence of $T$. gondii infection and high levels of anti-
T. gondii $\operatorname{IgG}$ antibodies in subjects drawn from the Detroit Neighborhood Health Study in the USA [14]. In a study of pregnant women, those with serological evidence of $T$. gondii exposure showed positive correlations between IgG levels and the profile of mood scales depression and anxiety subscales [16]. In a study of male rats with experimental infection with $T$. gondii, researchers found that infected rats with $T$. gondii cysts in the forebrain showed an increased anxiety-related behavior [17]. There are conflicting results about the association of $T$. gondii infection and depression. Duffy and coworkers found a relationship between chronic $T$. gondii infection, depression and dysphoric mood in a military veteran women population [13]. On the other hand, in a study in mice, reactivation of chronic $T$. gondii infection by an immunosuppressive regimen caused induction of depressive-like behaviors [18]. In contrast, in a meta-analysis of 50 studies reporting seroprevalence of T. gondii infection in any psychiatric disorder compared with control subjects, researchers found no association of anti- $T$. gondii antibodies with major depression [11]. Differences in the finding of an association between $T$. gondii infection and depression could be due to differences in the severity of depression among the studies. Depression in mixed anxiety and depressive disorder is mild. Therefore, it is likely that $T$. gondii infection can be rather associated with mild depression than with severe depression. Further studies including patients suffering from minor and major depression to elucidate the link of T. gondii exposure and depression should be conducted. Very little is known about the mechanisms that $T$. gondii uses to cause anxiety and depression. It is well known that $T$. gondii invades the brain [12], and this invasion might result in behavioral changes. Mood disorders associated with $T$. gondii can be due to changes in the concentrations of some neurotransmitters in brain including dopamine and serotonin [19]. In the present study, the association of $T$. gondii infection with mixed anxiety and depressive disorder was related to IgG seropositivity but not to IgM seropositivity. This finding suggests that this disorder is associated with latent infection rather than with acute infection.

We observed that the association of infection with $T$. gondii and mixed anxiety and depressive disorder was influenced by gender and age. A strong association was found in females and in patients aged 31 - 50 years old. It is not clear why the association was influenced by these gender and age groups. Seroprevalence of T. gondii infection in Durango is similar in male and females, and increases with age [15]. On the other hand, women suffer from anxiety and depression more frequently than men. In a recent study in a treatment-seeking population, women had a higher prevalence of anxiety disorders than men [20]. Similarly, in the population-based Hispanic Community Health Study/Study of Latinos in the USA, the prevalences of depression and anxiety were higher in women than in men, and in the age group of $45-64$ years than in the groups of $18-44$ years and 65 - 74 years [21]. Therefore, the fact that the association of $T$. gondii infection and mixed anxiety and depressive disorder was stronger in women and in people of middle age than in men and in younger and older age groups in our study further supports a contributing role of $T$. gondii infection and this disorder. Our findings have also epidemiological importance; if this association is confirmed in other studies, the ben- 
efit of treatment against $T$. gondii infection should be evaluated in patients suffering from anxiety and depressive disorder. $\mathrm{Pa}$ tients with bipolar disorder seropositive to $T$. gondii showed more depressive episodes when treated by drugs without anti$T$. gondii activity than patients who received drugs with anti- $T$. gondii activity [22].

A limitation of the present study was a small sample size of patients suffering from anxiety and depressive disorder. Further studies should have a larger sample size of patients.

\section{Conclusions}

Results support for the first time an association between seropositivity to $T$. gondii and mixed anxiety and depressive disorder. Further research to confirm this association and to determine the seroepidemiology of $T$. gondii infection in patients with this disorder is needed.

\section{Conflicts of Interest}

The authors declare that no conflict of interest exists.

\section{Grant Support}

This study was financially supported by Secretary of Public Education, Mexico (Grant No. DSA/103.5/14/11311).

\section{References}

1. Dubey JP. History of the discovery of the life cycle of Toxoplasma gondii. Int J Parasitol. 2009;39(8):877-882.

2. Schluter D, Daubener W, Schares G, Gross U, Pleyer U, Luder C. Animals are key to human toxoplasmosis. Int J Med Microbiol. 2014;304(7):917-929.

3. Montoya JG, Liesenfeld O. Toxoplasmosis. Lancet. 2004;363(9425):1965-1976.

4. Hide G, Morley EK, Hughes JM, Gerwash O, Elmahaishi MS, Elmahaishi KH, Thomasson D, et al. Evidence for high levels of vertical transmission in Toxoplasma gondii. Parasitology. 2009;136(14):1877-1885.

5. Derouin F, Pelloux H. Prevention of toxoplasmosis in transplant patients. Clin Microbiol Infect. 2008;14(12):1089-1101.

6. Figueroa Damian R. [Risk of transmission of infectious diseases by transfusion]. Ginecol Obstet Mex. 1998;66:277-283.

7. Maenz M, Schluter D, Liesenfeld O, Schares G, Gross U, Pleyer U. Ocular toxoplasmosis past, present and new aspects of an old disease. Prog Retin Eye Res. 2014;39:77106.

8. Machala L, Kodym P, Maly M, Geleneky M, Beran O, Jilich D. [Toxoplasmosis in immunocompromised patients]. Epidemiol Mikrobiol Imunol. 2015;64(2):59-65.

9. Flegr J. Schizophrenia and Toxoplasma gondii: an un- dervalued association? Expert Rev Anti Infect Ther. 2015;13(7):817-820.

10. Alvarado-Esquivel C, Urbina-Alvarez JD, EstradaMartinez S, Torres-Castorena A, Molotla-de-Leon G, Liesenfeld O, Dubey JP. Toxoplasma gondii infection and schizophrenia: a case control study in a low Toxoplasma seroprevalence Mexican population. Parasitol Int. 2011;60(2):151-155.

11. Sutterland AL, Fond G, Kuin A, Koeter MW, Lutter R, van Gool T, Yolken R, et al. Beyond the association. Toxoplasma gondii in schizophrenia, bipolar disorder, and addiction: systematic review and meta-analysis. Acta Psychiatr Scand. 2015;132(3):161-179.

12. Fond G, Capdevielle D, Macgregor A, Attal J, Larue A, Brittner M, Ducasse D, et al. [Toxoplasma gondii: a potential role in the genesis of psychiatric disorders]. Encephale. 2013;39(1):38-43.

13. Duffy AR, Beckie TM, Brenner LA, Beckstead JW, Seyfang A, Postolache TT, Groer MW. Relationship Between Toxoplasma gondii and Mood Disturbance in Women Veterans. Mil Med. 2015;180(6):621-625.

14. Markovitz AA, Simanek AM, Yolken RH, Galea S, Koenen KC, Chen S, Aiello AE. Toxoplasma gondii and anxiety disorders in a community-based sample. Brain Behav Immun. 2015;43:192-197.

15. Alvarado-Esquivel C, Estrada-Martinez S, Pizarro-Villalobos H, Arce-Quinones M, Liesenfeld O, Dubey JP. Seroepidemiology of Toxoplasma gondii infection in general population in a northern Mexican city. J Parasitol. 2011;97(1):40-43.

16. Groer MW, Yolken RH, Xiao JC, Beckstead JW, Fuchs D, Mohapatra SS, Seyfang A, et al. Prenatal depression and anxiety in Toxoplasma gondii-positive women. Am J Obstet Gynecol. 2011;204(5):433 e431-437.

17. Evans AK, Strassmann PS, Lee IP, Sapolsky RM. Patterns of Toxoplasma gondii cyst distribution in the forebrain associate with individual variation in predator odor avoidance and anxiety-related behavior in male LongEvans rats. Brain Behav Immun. 2014;37:122-133.

18. Mahmoud ME, Ihara F, Fereig RM, Nishimura M, Nishikawa Y. Induction of depression-related behaviors by reactivation of chronic Toxoplasma gondii infection in mice. Behav Brain Res. 2016;298(Pt B):125-133.

19. Henriquez SA, Brett R, Alexander J, Pratt J, Roberts CW. Neuropsychiatric disease and Toxoplasma gondii infection. Neuroimmunomodulation. 2009;16(2):122-133.

20. Pesce L, van Veen T, Carlier I, van Noorden MS, van der Wee NJ, van Hemert AM, Giltay EJ. Gender differences in outpatients with anxiety disorders: the Leiden Routine Outcome Monitoring Study. Epidemiol Psychiatr Sci. 2016;25(3):278-287.

21. Wassertheil-Smoller S, Arredondo EM, Cai J, Castaneda SF, Choca JP, Gallo LC, Jung M, et al. Depression, anxiety, antidepressant use, and cardiovascular disease among Hispanic men and women of different national backgrounds: results from the Hispanic Community Health Study/Study of Latinos. Ann Epidemiol. 2014;24(11):822-830.

22. Fond G, Boyer L, Gaman A, Laouamri H, Attiba D, 
Richard JR, Delavest M, et al. Treatment with anti-toxoplasmic activity (TATA) for toxoplasma positive patients with bipolar disorders or schizophrenia: a cross-sectional study. J Psychiatr Res. 2015;63:58-64. 